\title{
Lista de pareceristas ad hoc 2010/2011
}

\begin{abstract}
A Comissão Editorial de Política \& Sociedade agradece às pessoas abaixo, que colaboraram como pareceristas ad hoc no período 2010/2011.
\end{abstract}

Ademar Ribeiro Romeiro - Universidade Estadual de Campinas

Adriana Mohr - Universidade Federal de Santa Catarina

Adriano Naves de Brito - Universidade do Vale do Rio dos Sinos

Adriano Nervo Codato - Universidade Federal do Paraná

Agnaldo dos Santos - Universidade Estadual Paulista

Alberto Ribeiro Gonçalves de Barros - Universidade de São Paulo

Alexandre Braga Massella - Universidade de São Paulo

Alfredo José da Veiga-Neto - Universidade Federal do Rio Grande do Sul

Alícia Ferreira Gonçalves - Universidade Federal da Paraíba

Allene Carvalho Lage - Universidade Federal de Pernambuco

Ana Lúcia de Castro - Universidade Estadual Paulista

Ana Maria Kirschner - Universidade Federal Fluminense

Ana Rosa Cloclet da Silva - Pontifícia Universidade Católica de Campinas

Ana Selva Albinati - Pontifícia Universidade Católica de Minas Gerais

Anderson Moebus Retondar - Universidade Federal da Paraíba

André de Faria Pereira Neto - Fundação Oswaldo Cruz

André Pires - Pontifícia Universidade Católica de Campinas

Andrea Ribeiro Hoffmann - Universität Erfurt (Alemanha)

Andrei Koerner - Universidade Estadual de Campinas

Angela Cristina Salgueiro Marques - Universidade Federal de Minas Gerais

Angela Ganen - Universidade Federal Fluminense 
Angela Maria R. Paiva - Pontifícia Universidade Católica do Rio de Janeiro Antônia Colbari - Universidade Federal do Espírito Santo Antonio César Ortega - Universidade Federal de Uberlândia Antonio José Romera Valverde - Pontifícia Universidade Católica de São Paulo Ariane Patrícia Ewald - Universidade do Estado do Rio de Janeiro Arilson da Silva Favareto - Universidade Federal do ABC Armindo S. de Sousa Teodósio - Pontifícia Universidade Católica de Minas Gerais Arthur Trindade Maranhão Costa - Universidade de Brasília Ary Cesar Minella - Universidade Federal de Santa Catarina Atila Amaral Brilhante - Universidade Federal do Ceará Attila Magno e Silva Barbosa - Universidade Federal de Pelotas Belinda Pereira da Cunha - Universidade Federal da Paraíba Bruno Sciberras de Carvalho - Universidade Federal do Rio de Janeiro Bruno Wilhelm Speck - Universidade Estadual de Campinas Carlos Alfredo Gadea Castro - Universidade do Vale do Rio dos Sinos Carlos Antonio Giovinazzo Júnior - Pontifícia Universidade Católica de São Paulo Carlos E. Carvalho - Pontifícia Universidade Católica de São Paulo Carlos Eduardo de Freitas Vian - Universidade de São Paulo Carlos M. de Oliveira Junior - Pontifícia Universidade Católica do Rio de Janeiro Carlos Schmidt Arturi - Universidade Federal do Rio Grande do Sul Cesar Candiotto - Pontifícia Universidade Católica do Paraná Cesar Marcelo Baquero Jacome - Universidade Federal do Rio Grande do Sul Cezar Luiz de Mari - Universidade Federal de Viçosa Christian Edward Cyril Lynch - Universidade Gama Filho Clara Maria de Oliveira Araujo - Universidade do Estado do Rio de Janeiro Claudia Choma Bettega Almeida - Universidade Federal do Paraná Cláudio Gonçalves Couto - Fundação Getulio Vargas Claudio Roberto Amitrano - Instituto de Pesquisa Econômica Aplicada Clodoaldo Meneguello Cardoso - Universidade Estadual Paulista Cristiano Fonseca Monteiro - Universidade Federal Fluminense Cristiano Luis Lenzi - Universidade de São Paulo 
Déa de Lima Vidal - Universidade Estadual do Ceará

Deisimer Gorczevski - Universidade Federal do Ceará

Denise Barbosa Gros - Fundação de Economia e Estatística

Denise Paiva Ferreira - Universidade Federal de Goiás

Ednaldo Aparecido Ribeiro - Universidade Estadual de Maringá

Eduardo Rodrigues Gomes - Universidade Federal Fluminense

Eduardo Noronha - Universidade Federal de São Carlos

Elaine da Silveira Leite - Universidade Federal de São Carlos

Eliane Tomiasi Paulino - Universidade Estadual de Londrina

Elson Luciano Silva Pires - Universidade Estadual Paulista

Emerson Urizzi Cervi - Universidade Federal do Paraná

Enoque Ribeiro dos Santos - Universidade de São Paulo

Enrique Serra Padrós - Universidade Federal do Rio Grande do Sul

Erica Simone Almeida Resende - Universidade Federal Rural do Rio de Janeiro

Ernani Lampert - Universidade Federal do Rio Grande

Ester Vaisman - Universidade Federal de Minas Gerais

Fabiano Engelmann - Universidade Federal do Rio Grande do Sul

Fábio Luiz Búrigo - Universidade Federal de Santa Catarina

Fernanda Antonia da Fonseca Sobral - Universidade de Brasília

Fernando Antonio Farias de Azevedo - Universidade Federal de São Carlos

Fernando Augusto Starepravo - Universidade Estadual de Maringá

Fernando Dias de Avila-Pires - Fundação Oswaldo Cruz

Fernando Negret Fernandez - Faculdades Alves Faria

Flávia Millena Biroli Tokarski - Universidade de Brasília

Flávio Sacco dos Anjos - Universidade Federal de Pelotas

Francisco Luiz Corsi - Universidade Estadual Paulista

Gabriel de Santis Feltran - Universidade Federal de São Carlos

Geralda Luiza de Miranda - Instituto de Pesquisa Econômica Aplicada

Geraldo Augusto Pinto - Universidade Estadual do Oeste do Paraná

Guilherme G. Freitas Xavier Sobrinho - Fundação de Economia e Estatística 
Gustavo Ferreira da Costa Lima - Universidade Federal da Paraíba Helcimara de Souza Telles - Universidade Federal de Minas Gerais Hélio Rebello Cardoso Jr - Universidade Estadual Paulista Humberto Eduardo de Paula Martins - Universidade Federal de Uberlândia Igor Gastal Grill - Universidade Federal do Maranhão Ilse Scherer-Warren - Universidade Federal de Santa Catarina Inês Emília de Moraes Sarmento Patrício - Universidade Federal Fluminense Islandia Bezerra da Costa - Universidade Federal do Paraná Ivo José de Aquino Coser - Universidade Federal do Rio de Janeiro Jacques Mick - Universidade Federal de Santa Catarina Jairo Cesar Marconi Nicolau - Instituto de Estudos Sociais e Políticos Jandir Ferrera de Lima - Universidade Estadual do Oeste do Paraná Janice Tirelli Ponte de Sousa - Universidade Federal de Santa Catarina Janina Onuki - Universidade de São Paulo Jessie Jane Vieira de Sousa - Universidade Federal do Rio de Janeiro Jesus José Ranieri - Universidade Estadual de Campinas João Batista de Jesus Felix - Fundação Universidade Federal do Tocantins João Carlos Salles Pires da Silva - Universidade Federal da Bahia João Luiz Medeiros - Montpellier (França) - Ecole des Hautes Etudes de Journalisme

João Marcelo Ehlert Maia - Fundação Getúlio Vargas

João Vicente R. Barroso da Costa Lima - Universidade Federal de Santa Maria John P. Woodall - Universidade Federal do Rio de Janeiro Jose Raymundo Novaes Chiappin - Universidade de São Paulo José Ricardo Garcia Pereira Ramalho - Universidade Federal do Rio de Janeiro Juliana Santos Maia Bertazzo - Universidade Estadual de Campinas Jussara Reis Prá - Universidade Federal do Rio Grande do Sul Karine Pereira Goss - Instituto Federal de Educação, Ciência e Tecnologia Leila de Menezes Stein - Universidade Estadual Paulista Leopoldo Garcia Pinto Waizbort - Universidade de São Paulo 
Lucia Helena Alves Müller - Pontifícia Universidade Católica do Rio Grande do Sul Lucia Helena de Oliveira Cunha - Universidade Federal da Paraíba Luciana Beatriz de Oliveira Bar de Carvalho - Universidade de Uberaba Luciana Ferreira Tatagiba - Universidade Estadual de Campinas Luciano Aronne de Abreu - Pontifícia Universidade Católica do Rio Grande do Sul Luciano Félix Florit - Fundação Universidade Regional de Blumenau Luis Felipe Miguel - Universidade de Brasília Luís Mauro Sá Martino - Faculdade Cásper Líbero Luiz Claudio Lourenço - Universidade Federal da Bahia Manuel Domingos Neto -Universidade Federal Fluminense Marcelo Domingos Sampaio Carneiro - Universidade Federal do Maranhão Marcelo Kunrath Silva - Universidade Federal do Rio Grande do Sul Marcelo Pereira de Mello - Universidade Federal Fluminense Marcelo Siqueira Ridenti - Universidade Estadual de Campinas Marcia Cristina Consolim - Universidade Federal de São Paulo Márcia da Silva Mazon - Universidade Federal de Santa Catarina Márcia Grisotti - Universidade Federal de Santa Catarina Márcio Sergio Batista Silveira de Oliveira - Universidade Federal do Paraná Marcos Tadeu Del Roio - Universidade Estadual Paulista Maria Antônia de Souza - Universidade Tuiuti do Paraná Maria da Penha Smarzaro Siqueira - Universidade Federal do Espírito Santo Maria de Lurdes Costa Domingos - Universidade Católica de Petrópolis Maria do Socorro Sousa Braga - Universidade Federal de São Carlos Maria Lucia Caira Gitahy - Universidade de São Paulo Maria Salete Souza de Amorim - Universidade Federal da Bahia Maria Soledad Etcheverry Orchard - Universidade Federal de Santa Catarina Maria Teresa Miceli Kerbauy - Universidade Estadual Paulista Marilene de Souza Campos - Universidade Federal de Viçosa Marina de Souza Sartore - Universidade Federal de Goiás Mauricio Roque Serva de Oliveira - Universidade Federal de Santa Catarina 
Monica Hass - Universidade Federal da Fronteira Sul

Mônica Landi - Pontifícia Universidade Católica de São Paulo

Nelson E. Pinheiro Montenegro - Universidade Federal do Recôncavo da Bahia

Nelson Giordano Delgado - Universidade Federal Rural do Rio de Janeiro

Nildo Silva Viana - Universidade Federal de Goiás

Odaci Luiz Coradini - Universidade Federal do Rio Grande do Sul

Osmir Dombrowski - Universidade Estadual do Oeste do Paraná

Oswaldo Dehon Roque Reis - Instituto Brasileiro de Mercado de Capitais

Otavio Soares Dulci - Universidade Federal de Minas Gerais

Paola Cappellin - Universidade Federal do Rio de Janeiro

Patricia Caldeira - Faculdade de Direito de São Bernardo do Campo

Patricia Fontoura Aranovich - Universidade Federal de São Paulo

Patricia Rodrigues Chaves da Cunha - Universidade Federal de Pelotas

Pedro José Floriano Ribeiro - Universidade Federal de São Carlos

Pedro Roberto Jacobi - Universidade de São Paulo

Philippe Pomier Layrargues - Universidade de Brasília

Raquel Kritsch - Universidade Estadual de Londrina

Rachel Soihet - Universidade Federal Fluminense

Raúl Enrique Rojo - Universidade Federal do Rio Grande do Sul

Rebecca Neaera Abers - Universidade de Brasília

Renato Raul Boschi - Instituto de Estudos Sociais e Políticos

Ricardo Abramovay - Universidade de São Paulo

Ricardo Fabrino Mendonça - Universidade Federal de Minas Gerais

Roberto Grün - Universidade Federal de São Carlos

Roberto Rocha Coelho Pires - Instituto de Pesquisa Econômica Aplicada

Rodrigo Constante Martins - Universidade Federal de São Carlos

Rodrigo Duarte Fernandes dos Passos - Universidade Federal do Piauí

Rodrigo Ghiringhelli de Azevedo - Pontifícia Universidade Católica do Rio Grande do Sul

Ruben George Oliven - Universidade Federal do Rio Grande do Sul 
Rubens Rogério Sawaya - Pontifícia Universidade Católica de São Paulo Rurion Soares Melo - Universidade Federal de São Paulo Salvador André Schavelzon - Pontifícia Universidade Católica de Campinas Samuel Alves Soares - Universidade Estadual Paulista

Selene de Souza C. Herculano dos Santos - Universidade Federal Fluminense Silke Weber - Universidade Federal de Pernambuco

Silvia Mello Souto Maior - Instituto Federal de Educação

Sílvio Cário - Universidade Federal de Santa Catarina

Silvio César Camargo - Universidade Estadual de Campinas

Silvio Segundo Salej Higgins - Universidade Federal de Minas Gerais

Simone Rodriguez Bohn - Toronto (Canadá) - York University

Sônia Guimarães - Universidade Federal do Rio Grande do Sul

Tamara Benakouche - Universidade Federal de Santa Catarina

Tiago Bahia Losso - Universidade Federal de Santa Catarina

Valdemir Aparecido Pires - Universidade Estadual Paulista

Vanessa Elias de Oliveira - Universidade Federal do ABC

Vera Alves Cepêda - Universidade Federal de São Carlos

Verônica Morais Ximenes - Universidade Federal do Ceará

Vitor de Moraes Peixoto - Universidade Estadual do Norte Fluminense

Vitor Emanuel Marchetti Ferraz Junior - Universidade Federal do ABC

Viviane Melo de Mendonça-Magro -Universidade Federal de São Carlos

Wagner Pralon Mancuso - Universidade de São Paulo

Wagner Tadeu Iglecias - Universidade de São Paulo

Wanderley Marchi Júnior - Universidade Federal do Paraná

Wânia Pasinato - Universidade de São Paulo

Weimar Freire da Rocha Jr - Universidade Estadual do Oeste do Paraná

Williams da Silva Gonçalves - Universidade do Estado do Rio de Janeiro

Zander Soares de Navarro - Universidade Federal do Rio Grande do Sul 